\title{
The Information-Theoretic Treatment of Spinless Particles with the Assorted Diatomic Molecular Potential
}

\author{
Muhammad Roshanzamir \\ Division of Elementary Particles and Field Theory, Department of Physics, Faculty of Basic Sciences, Shahrekord University, Iran \\ Correspondence should be addressed to Muhammad Roshanzamir; m.roshanzamir@sku.ac.ir
}

Received 27 December 2020; Revised 24 September 2021; Accepted 22 December 2021; Published 18 January 2022

Academic Editor: Elias C. Vagenas

Copyright (C) 2022 Muhammad Roshanzamir. This is an open access article distributed under the Creative Commons Attribution License, which permits unrestricted use, distribution, and reproduction in any medium, provided the original work is properly cited.

\begin{abstract}
The relativistic solutions of the Klein-Gordon equation comprising an interaction of the generalized inversely quadratic Yukawa potential mixed linearly with the hyperbolic Schiöberg molecular potential is achieved employing the idea of parametric Nikiforov-Uvarov and the Greene-Aldrich approximation scheme. The energy spectra and the corresponding normalized wave functions are derived regarding the hypergeometric function in a closed form for arbitrary $\ell$-state. Numerical results of the energy eigenvalue are proposed. Moreover, special circumstances of this potential are reviewed, and their energy eigenvalues were assessed. Subsequently, the Tsallis entropy and Rényi entropy both in position and momentum spaces are defined under the desired potential. The impacts of these entropies on the angular momentum quantum number are explored in detail.
\end{abstract}

\section{Introduction}

In recent years, different researches have centered on the investigations of the quantum mechanical issues with various physical potentials because they incorporate all of the basic data required to correctly characterize physical quantum models [1-3]. It is worthy of note that the investigation of the relativistic wave equation especially the Klein-Gordon equation has raised fundamentally more thought of numerous researchers as well as its considerable impact in quantum mechanics. These equations may explicitly predict the particles' treatment, which lead to the study of relativistic effects in various branches of physics and chemistry $[4,5]$. The Klein-Gordon equation is a notable relativistic wave equation that depicts the movement of spinless particles because of its square terms [6-8]. It is also realized that the specific feasible actual possibilities via the Klein-Gordon equations are uncommon, with the exception of some outstanding solvable quantum systems, for example, the hydrogen atom and harmonic oscillator, while the case of states with arbitrary angular momentum, which do not exhibit accurate solutions, is understood either mathematically or by approximation methods $[9,10]$. In this manner, numer- ous quantum modes should be considered by approximation schemes, and the decision of these utilized approximation layouts relies upon their adaptability to the desired potential $[11,12]$. Because of their various applications, solutions of the relativistic and nonrelativistic wave equations have been utilized in various quantum potential interactions utilizing different techniques $[13,14]$. These methods incorporate $1 /$ $N$ shifted expansion procedure [15], Nikiforov-Uvarov approach [16, 17], asymptotic iteration method [18], factorization method $[19,20]$, formula technique [21], supersymmetric approach $[22,23]$, ansatz methodology [24], Laplace transform approach $[25,26]$, functional analysis approach $[27,28]$, proper quantization rule [29], and others [30, 31].

The hypergeometric Nikiforov-Uvarov method has indicated its capacity to ascertain the specific energy levels of bound states for many solvable quantum frameworks [32]. The parametric adaptation of this approach is employed to acquire the bound-state solutions of the $\mathscr{D}$-dimensional radial Klein-Gordon equation [33]. It is supported by its acceptable execution through comparisons with various techniques and a direct, precise, and user-friendly approach.

Using exponential-type potentials, various quantum mechanical wave equations have been extensively studied 
$[34,35]$. It has been contended that these types of potentials are much more efficient than their competitors, which exist in Coulomb, or inverse forms and have significant roles in solid-state physics, nuclear physics, and other fascinating domains $[36,37]$. In the study of potentials with hypergeometric wave functions, a suitable form of a composed exponential-type potential is presumed, which grants obtaining various exponential potentials. Such potentials can be comprehensively utilized as interaction models of diatomic molecules. The desired combined potential encompasses the generalized inversely quadratic Yukawa potential with a molecular hyperbolic potential, which is given by

$$
\begin{aligned}
V_{\text {SGIQYP }}= & -T e^{-2 \sigma r}\left(\frac{2}{r^{2}}+2\left(-\frac{2 Q}{r}+Q^{2} e^{\sigma r}\right) e^{\sigma r}\right) \\
& +\frac{2 H\left(\mathrm{Q}\left(e^{2 \sigma r}+1\right)-e^{2 \sigma r}+1\right)^{2}}{\left(e^{2 \sigma r}-1\right)^{2}},
\end{aligned}
$$

where $T, \sigma$, and $Q$ are the coupling strength, the screening parameter, and a constant (an inverse length), respectively, where $\sigma<1$ and $H$ along with $\varrho$ are two adjustable positive parameters representing the properties of the potential where $0 \leq \mathrm{Q} \leq 1$.

This combination might describe the effective potential of a many-body system since it incorporates the notable potential models as special cases. This model can be utilized in a variety of fields such as atomic, solid-state, plasma, and quantum field theory, where it has a minimum value 0 and can attain infinity when $r=0$. Hence, this potential represents better treatment than other exponential potentials in some cases and is able to modify comparatively the efficiency of other potentials. In this manner, the study of quantum systems linked with these forms of potentials seems substantial in proposing several frameworks for quantum chemistry and molecular physics. The current investigation was inspired by the idea that this type of potential could be used as a mathematical example to impressively depict the characteristics of diatomic molecules, despite the fact that there has been little research into this linear combination of diatomic molecular potential energy functions, particularly within the framework of the Klein-Gordon equation. Subsequently, the accomplishment of quantum mechanics in the depiction of the physics of atomic and subatomic particles cannot be overstated [13, 36, 38-42].

Spin and pseudo-spin symmetry have made a profit in recent studies due to their applications in nuclear physics and other related areas [43-46]. Recently, a large number of studies have demonstrated their advantages in acquiring the solutions of the Dirac equations for various generic potentials within the spin and pseudo-spin symmetric limit [47-52], but only a few investigations have been conducted within the framework of the Klein-Gordon structure. The objective of this research is to figure out the energy spectrum and wave function visualization for the Klein-Gordon equation at the spin symmetry limit using the Nikiforov-Uvarov technique and the Greene-Aldrich approximation scheme.

In this context, what is required in this paper is categorized as follows. In Section 2, the parametric Nikiforov-
Uvarov method for a generally second-order differential equation is briefly discussed. Section 3 employs the basic concept of the parametric Nikiforov-Uvarov approach via the Greene-Aldrich approximation scheme to obtain $\ell$ -wave approximate analytical solutions of the $\mathscr{D}$-dimensional radial Klein-Gordon equation and the normalized radial wave functions in terms of generalized hypergeometric functions for the desired potential within the spin symmetry limit. Entropic measures propose well-organized implementations to smooth the path for realizing connections in quantum frameworks [53-55]. Two basic theoretic quantities, such as the Rényi entropy $[56,57]$ and the Tsallis entropy $[58,59]$, are commonly used in information theory as a measure of entanglement. They have numerous purposes in optical lattice theory, statistical mechanics, and various realms of mathematics and physics and are calculated in Section 4. Section 5 is devoted to a brief discussion, while the conclusions are outlined in the last section.

\section{Parametric Nikiforov-Uvarov Methodology}

A numerous category of differential equations can be solved by the Nikiforov-Uvarov approach in mathematical physics, which relies on attenuating a second-order linear differential equation to a generalized equation of hypergeometric type [16]. Many accomplishments have been documented in the successful application of this technique to obtain viable results for various wave equations in a quantum framework $[60,61]$. The parametric form of this approach was introduced in order to have an efficient application [17] and can easily solve any differential equations of the form

$$
\left(\frac{d^{2}}{d p^{2}}++\frac{\omega_{1}-\omega_{2} p}{p\left(1-\omega_{3} p\right)} \frac{d}{d p}-\frac{1}{\left(p\left(1-\omega_{3} p\right)\right)^{2}}\left(\boldsymbol{g}_{1} p^{2}-\boldsymbol{g}_{2} p+\boldsymbol{g}_{3}\right)\right) \psi=0
$$

According to this approach, the eigenfunctions are expressed as

$$
\psi(p)=\mathcal{N}_{n, \ell} p^{\omega_{12}}\left(1-\omega_{3} p\right)^{-\omega_{12}-\omega_{13} / \omega_{3}} \mathscr{P}_{n}^{\left(\omega_{10}-1, \omega_{11} / \omega_{3}-\omega_{10}-1\right)}\left(1-2 \omega_{3} p\right),
$$

where $\mathscr{P}_{n}^{(\alpha, \beta)}(x)$ and $\mathcal{N}_{n, \ell}$ are the orthogonal Jacobi polynomial [62] and the normalization constant, respectively. Furthermore, the parametric energy eigenvalues are given as

$$
\begin{aligned}
& \omega_{2} n-(2 n+1) \omega_{5}+(2 n+1)\left(\sqrt{\omega_{9}}+\omega_{3} \sqrt{\omega_{8}}\right)+n(n+1) \omega_{3} \\
& \quad+2 \omega_{3} \omega_{8}+2 \sqrt{\omega_{8} \omega_{9}}+\omega_{7}=0,
\end{aligned}
$$


where

$$
\begin{gathered}
\omega_{4}=\frac{1}{2}\left(1-\omega_{1}\right), \\
\omega_{5}=\frac{1}{2}\left(\omega_{2}-2 \omega_{3}\right), \\
\omega_{6}=\omega_{5}^{2}+g_{1}, \\
\omega_{7}=2 \omega_{4} \omega_{5}-g_{2}, \\
\omega_{8}=\omega_{4}^{2}+g_{3}, \\
\omega_{9}=\omega_{3} \omega_{7}+\omega_{3}^{2} \omega_{8}+\omega_{6}, \\
\omega_{10}=\omega_{1}+2 \omega_{4}+2 \sqrt{\omega_{8}}, \\
\omega_{11}=\omega_{2}-2 \omega_{5}+2\left(\sqrt{\omega_{9}}+\omega_{3} \sqrt{\omega_{8}}\right), \\
\omega_{12}=\omega_{4}+\sqrt{\omega_{8}}, \\
\omega_{13}=\omega_{5}-\left(\sqrt{\omega_{9}}+\omega_{3} \sqrt{\omega_{8}}\right) .
\end{gathered}
$$

\section{D-Dimensional Klein-Gordon Equation}

The Klein-Gordon equation with a scalar potential $S(r)$ and a vector potential $V(r)$ in $\mathscr{D}$-dimensions is given by $[63,64]$

$$
\left(\hbar^{2} c^{2} \nabla_{\mathscr{D}}^{2}-\left(\mu c^{2}+S(r)\right)^{2}+\left(E_{n, \ell}-V(r)\right)^{2}\right) \Psi\left(r, \Omega_{\mathscr{D}}\right)=0,
$$

where $\nabla_{\mathscr{D}}^{2}$ represents the Laplace operator in $\mathscr{D}$-dimensions and is defined as

$$
\nabla_{\mathscr{D}}^{2}=\frac{1}{r^{\mathscr{D}-1}} \frac{\partial}{\partial r}\left(r^{\mathscr{D}-1} \frac{\partial}{\partial r}\right)-\frac{1}{r^{2}} K_{\mathscr{D}}^{2}\left(\Omega_{\mathscr{D}}\right)
$$

where $\mathscr{D}$ represents the spatial dimensionality where $\mathscr{D}$ $\geq 2, \hbar$ is the reduced Planck constant, and $\mu, c$, and $E_{n, \ell}$ are the rest mass of the particle, the speed of light, and the relativistic energy of the system, respectively. The attractive scalar potential is usually observed under static field conditions, whereas the repulsive vector potential is observed under dynamic conditions. The scalar potential $S(r)$ is regarded as an extra quantity added to the particle mass $\mu$. Also, the total wave function in $\mathscr{D}$-dimension can be given as $\Psi\left(r, \Omega_{\mathscr{D}}\right)=r^{1-\mathscr{D} / 2} U_{n, \ell}(r) Y_{\ell, m}\left(\Omega_{\mathscr{D}}\right)$, where $Y_{\ell, m}\left(\Omega_{\mathscr{D}}\right)$ is the generalized spherical harmonic function. Employing the eigenvalues of the generalized angular momentum operator, $K_{\mathscr{D}}^{2}\left(\Omega_{\mathscr{D}}\right)$, where $K_{\mathscr{D}}^{2}\left(\Omega_{\mathscr{D}}\right) Y_{\ell, m}\left(\Omega_{\mathscr{D}}\right)=\ell(\ell+\mathscr{D}-2) Y_{\ell, m}$ $\left(\Omega_{\mathscr{D}}\right)$, the radial part of the $\mathscr{D}$-dimensional Klein-Gordon Equation (6) is written as

$$
\begin{aligned}
& \left(\hbar^{2} c^{2} \frac{d^{2}}{d r^{2}}+\left(E_{n, \ell}^{2}-\mu^{2} c^{4}\right)-2\left(\mu c^{2} S(r)+E_{n, \ell} V(r)\right)+V^{2}(r)\right. \\
& \left.-S^{2}(r)-\frac{\wp(\wp+1) \hbar^{2} c^{2}}{r^{2}}\right) U_{n, \ell}(r)=0,
\end{aligned}
$$

where $\wp=\ell+\mathscr{D}-3 / 2$, and $\ell$ represents the angular momentum quantum number. With the equal scalar and vector potentials being taken as the determined potential Equation (1), where $Q=1$, the following second-order Schrödingerlike equation may be obtained as

$$
\begin{aligned}
& \left(\frac{d^{2}}{d r^{2}}+\frac{\left(E_{n, \ell}^{2}-\mu^{2} c^{4}\right)}{\hbar^{2} c^{2}}+\frac{2\left(\mu c^{2}+E_{n, \ell}\right)}{\hbar^{2} c^{2}}\left(\frac{T}{r^{2}} e^{-2 \sigma r}-\frac{2 T}{r} e^{-\sigma r}\right.\right. \\
& \left.\left.+T-\frac{H\left(\mathrm{\varrho}\left(e^{2 \sigma r}+1\right)-e^{2 \sigma r}+1\right)^{2}}{\left(e^{2 \sigma r}+1\right)^{2}}\right)-\frac{\wp(\wp+1)}{r^{2}}\right) U_{n, \ell}(r)=0,
\end{aligned}
$$

which cannot be solved analytically even for the $s$-wave case. In order to solve Equation (9) for arbitrary $\ell$-state and also to consider the Columbic characteristic $(1 / r)$ of the Yukawa potential, the following Greene and Aldrich approximation scheme for $\sigma \ll 1$ might be used as $[10,65]$

$$
\frac{1}{r^{2}} \approx \frac{4 \sigma^{2} e^{-2 \sigma r}}{\left(1-e^{-2 \sigma r}\right)^{2}}
$$

As comparison, the behavior of $1 / r^{2}$ and the recommended approximate term are shown in Figure 1. It is obvious that this approximation is most suitable for the low values of $\sigma$ that are considered in this work. Consequently, this approximation will be used throughout the present study.

Substituting Equation (10) into Equation (9) and introducing coordinate transformation of the form $h=e^{-2 \sigma r}$, the generalized hypergeometric-type equation reads as

$$
\frac{d^{2} U_{n, \ell}(h)}{d h^{2}}+\frac{1}{h} \frac{d U_{n, \ell}(h)}{d h}+\frac{1}{h^{2}(1-h)^{2}}\left(-\boldsymbol{g}_{1} h^{2}+\boldsymbol{g}_{2} h-\boldsymbol{g}_{3}\right) U_{n, \ell}(h)=0,
$$

where

$$
\begin{aligned}
& g_{1}= \frac{\left(-4 T \sigma^{2}-4 T \sigma+(\mathrm{Q}+1)^{2} H+1 / 2\left(c^{2} \mu-E_{n, \ell}\right)-T\right)\left(c^{2} \mu+E_{n, \ell}\right)}{2 c^{2} \hbar^{2} \sigma^{2}}, \\
& \boldsymbol{g}_{2}= \frac{1}{2 c^{2} \hbar^{2} \sigma^{2}}\left(c^{4} \mu^{2}+\left(-2 \wp \hbar^{2}(\wp+1) \sigma^{2}-4 T \mu \sigma-2 \mu\left(\mathrm{Q}^{2} H-H+T\right)\right) c^{2}\right. \\
&\left.-2 E_{n, \ell}\left(\mathrm{Q}^{2} H+2 T \sigma-H+\frac{E_{n, \ell}}{2}+T\right)\right), \\
& \boldsymbol{g}_{3}=\left((\mathrm{Q}-1)^{2} H+1 / 2\left(c^{2} \mu-E_{n, \ell}\right)-T\right)\left(c^{2} \mu+E_{n, \ell}\right) \\
& 2 c^{2} \hbar^{2} \sigma^{2}
\end{aligned}
$$

By comparing Equation (11) with Equation (2), one can obtain the following parametric coefficients as

$$
\omega_{1}=\omega_{2}=\omega_{3}=1,
$$




$$
\begin{aligned}
\omega_{4} & =0, \omega_{5}=-\frac{1}{2}, \omega_{6}=\frac{1}{4}+\boldsymbol{g}_{1}, \omega_{7}=-\boldsymbol{g}_{2}, \omega_{8}=\boldsymbol{g}_{3}, \omega_{9} \\
& =\boldsymbol{g}_{1}-\boldsymbol{g}_{2}+\boldsymbol{g}_{3}+\frac{1}{4}, \omega_{10}=1+2 \sqrt{\boldsymbol{g}_{3}}, \omega_{11} \\
& =2\left(1+\sqrt{\boldsymbol{g}_{3}}+\sqrt{\boldsymbol{g}_{1}-\boldsymbol{g}_{2}+\boldsymbol{g}_{3}+\frac{1}{4}}\right), \omega_{12}=\sqrt{\boldsymbol{g}_{3}}, \omega_{13} \\
& =-\frac{1}{2}-\sqrt{\boldsymbol{g}_{3}}-\sqrt{\boldsymbol{g}_{1}-\boldsymbol{g}_{2}+\boldsymbol{g}_{3}+\frac{1}{4}} .
\end{aligned}
$$

The D-dimensional relativistic energy spectra for the Schiöberg-generalized inversely quadratic Yukawa potential model can be obtained explicitly by substituting Equation (13) into Equation (4) as

$$
\begin{aligned}
& \frac{1}{2 c^{2} \hbar^{2} \sigma^{2}}\left(\left(\sqrt{\mu c^{2}+E_{n, \ell}} \sqrt{2}(\varrho-1)^{2} H+\mu c^{2}-\left(E_{n, \ell}+2 T\right)+2 \sigma\left(n+\frac{1}{2}\right) c \hbar\right)\right. \\
& \quad \times \sqrt{\left(\left(4\left(\wp+\frac{1}{2}\right)^{2} \hbar^{2}-8 T \mu\right) c^{2}-8 E_{n, \ell} T\right) \sigma^{2}+8 \varrho^{2}\left(\mu c^{2}+E_{n, \ell}\right) H} \\
& \quad+2 \sigma\left(n+\frac{1}{2}\right) \hbar c \sqrt{\mu c^{2}+E_{n, \ell}} \sqrt{2(\varrho-1)^{2} H+\mu c^{2}-E_{n, \ell}-2 T} \\
& +\left(2\left(\wp(\wp+1)+n(n+1)+\frac{1}{2}\right) \hbar^{2} \sigma^{2}+4 \mu(T \sigma+H \varrho(\varrho-1))\right) c^{2} \\
& \left.\quad+4 E_{n, \ell}(T \sigma+H \varrho(\varrho-1))\right)=0 .
\end{aligned}
$$

In addition, the corresponding wave function for the proposed potential is obtained by substituting Equation (13) into Equation (3) as

$$
U_{n}(h)=\mathcal{N}_{n} h^{\zeta}(1-h)^{\rho} P_{n}^{(2 \zeta, 2 \rho-1)}(1-2 h),
$$

where

$$
\begin{gathered}
\zeta=\frac{\sqrt{\left(\mu c^{2}-E_{n, \ell}+2(\varrho-1)^{2} H-2 T\right)\left(\mu c^{2}+E_{n, \ell}\right)}}{2 c \hbar \sigma}, \\
\rho=\frac{c \hbar \sigma+\sqrt{\left(\left(4(\wp+1 / 2)^{2} \hbar^{2}-8 T \mu\right) c^{2}-8 E_{n, \ell} T\right) \sigma^{2}+8 \mathrm{Q}^{2}\left(\mu c^{2}+E_{n, \ell}\right) H}}{2 c \hbar \sigma},
\end{gathered}
$$

where $P_{n}^{(\chi, \varsigma)}$ denotes the Jacobi polynomials, which have been used extensively in mathematical analysis and practical applications. Using the orthogonality property of the Jacobi polynomials [62],

$\int_{-1}^{1} \mathscr{P}_{a}^{(\chi, \varsigma)} \mathscr{P}_{b}^{(\chi, \varsigma)}(1-\varpi)^{\chi}(1+\varpi)^{\varsigma} d x=\frac{2^{\chi+\varsigma+1}}{2 n+\chi+\varsigma+1} \frac{\Gamma(n+\chi+1) \Gamma(n+\varsigma+1)}{n ! \Gamma(n+\chi+\varsigma+1)} \delta_{a b}$,

and the normalization coefficient $\mathcal{N}_{n}$ can be expressed as

$$
\mathcal{N}_{n}=\left(\frac{\zeta \sigma(\rho+n+\zeta) \Gamma(n+1) \Gamma(\rho+n+2 \zeta-1)}{(n+\rho) \Gamma(n+2 \rho)}\right)^{1 / 2}
$$

The solution of the Schrödinger equation can be obtained directly from the solution of the relativistic KleinGordon equation by making some certain transformations. Thus, employing the following mapping within the nonrelativistic limits [25], where $\sigma<1$ and $0 \leq \mathrm{Q} \leq 1$,

$$
\begin{gathered}
\mu c^{2}+E_{n, \ell} \longrightarrow 2 \mu c^{2}, \\
E_{n, \ell}-\mu c^{2} \longrightarrow E_{n, \ell}{ }^{\text {N.R. }},
\end{gathered}
$$

the corresponding $\mathscr{D}$-dimensional nonrelativistic energy relation for the potential model Equation (1) is attained as

$$
\begin{aligned}
E_{n J}^{\text {N.R. }}= & -2\left(\hbar^{2}\left(\wp(\wp+1)+n(n+1)+\frac{1}{2}\right) \sigma^{2}+\left(4 T \mu+\left(n+\frac{1}{2}\right) \hbar\right.\right. \\
& \left.\left.\times \sqrt{\left(4\left(\wp+\frac{1}{2}\right)^{2} \hbar^{2}-16 \mu T\right) \sigma^{2}+16 \mathrm{Q}^{2} H \mu}\right) \sigma+4 \mathrm{\varrho} \mu H(\mathrm{\varrho}-1)^{2}\right)^{2} \\
& \cdot\left(\left(\hbar \sigma(2 n+1)+\sqrt{\left(4\left(\wp+\frac{1}{2}\right)^{2} \hbar^{2}-16 \mu T\right) \sigma^{2}+16 \mathrm{Q}^{2} H \mu}\right)^{2} \mu\right)^{-1} \\
& +2\left((\varrho-1)^{2} H-T\right) .
\end{aligned}
$$

\section{Information-Theoretic Measures}

The concept of information theory plays a significant role in contemporary theoretical physics, while it has not been much discussed in the current research union. As has been demonstrated, the information-theoretic technique, which offers novel subjects, proposes wide insight into many other physical problems $[66,67]$. The use of these problem-solving viewpoints, which best characterize the energy characterisation of these systems, exposes the information-based demonstration of molecular states to debate. According to statistical mechanics, the entropic uncertainty relation operates similarly to the Heisenberg uncertainty [68]. Information-theoretic measures have been used to explore numerous unique distributions that define the quantum mechanical states of various possible models [69]. Among them, the Rényi entropy $[56,68]$ and the Tsallis entropy $[59,68]$ via the given potential are the subjects of our study in this paper. It is known that suggested entropies in the position space represent the uncertainties in the localization of a particle in space, whereas the momentum space entropies measure the uncertainties in estimating the momentum of the particle. These entropies are tightly related to basic and experimentally measurable variables like kinetic energy and magnetic susceptibility, making them useful in studying the structure and dynamics of atomic and molecular systems [70]. One of the primary distinctions is that the Rényi entropy is additive, whereas the Tsallis entropy is nonadditive (or nonextensive). Supplementary comparison analyses may be found, for example, in [70] between these entropies.

4.1. Rényi Entropy. As a beneficial extension of the Shannon entropy, the Rényi entropy [56] was offered by Rényi in 1960 as a class of information-theoretic measures, which included many of its features and demonstrated numerous purposes. 


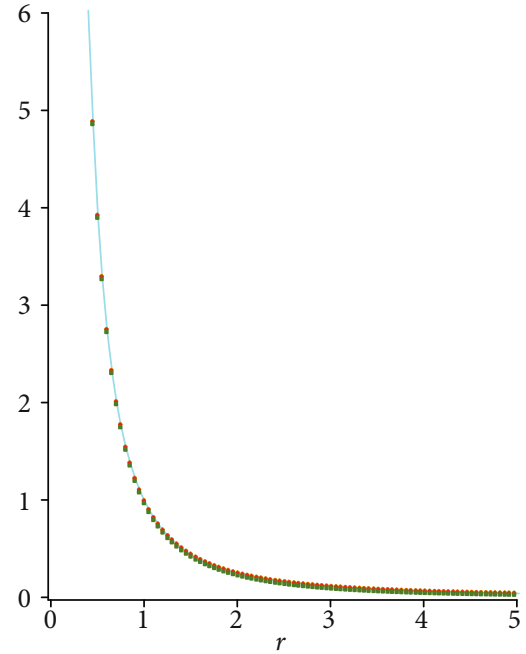

$$
\begin{aligned}
& -\frac{1}{r^{2}} \\
& \text { - } \sigma=0.01 \\
& \sigma=0.05 \\
& \sigma=0.1 \\
& \text { - } \sigma=0.3
\end{aligned}
$$

FIGURE 1: Plot of $1 / r^{2}$ and its approximation in Equation (10) with $\sigma=0.01,0.05,0.1$, and 0.3 .

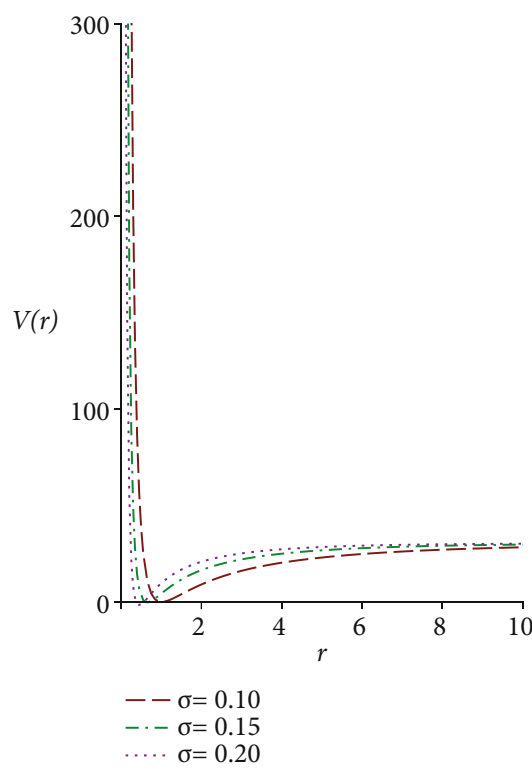

FIGURE 2: Sketch of the composed-potential as a function of $r$ $(r>0)$ for $H=20, T=1$, and $\mathrm{\varrho}=0.1$ with $\sigma=0.1,0.15$, and 0.2 .

Its applications in physics include, but are not limited to, in, quantum entanglement [71, 72], quantum communication protocols [73], and quantum measurement [74]. Applying the transformation $h=e^{-2 \sigma r}$, the Rényi entropy is given as [56]

$$
S_{\eta}^{\left(\mathscr{R}_{p}\right)}(\delta)=\frac{1}{\eta-1} \operatorname{Ln} \frac{2 \pi}{\sigma} \int_{1}^{0} \delta(h)^{\eta} \frac{1}{h} d h,
$$

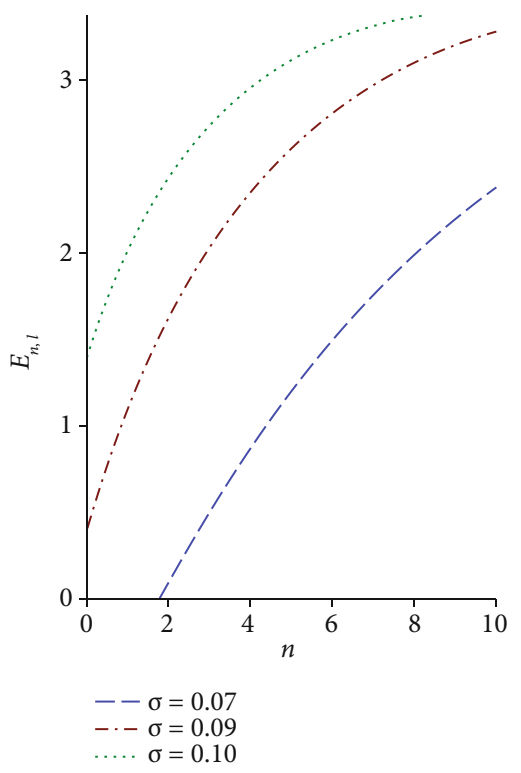

FIgURE 3: Behavior of energy spectral with respect to $n$ with $\mu=c$ $=\hbar=1, H=20, \varrho=0.1, T=15$, and $\mathscr{D}=5$ for $\sigma=0.07,0.09$, and 0.1 .

where $\eta$ denotes the entropic index. The Rényi entropy measures the sensitivity of the system to the deviation from the equilibrium. If the $\eta$ parameter is larger than unity, the related entropy decreases, implying that the object is provided with more information on such a configuration than its equilibrium equivalent. The rate of change of the entropy with the Rényi parameter simply illustrated the sensitivity of the system to the degree of nonequilibricity. The Rényi entropy in the position space is mathematically specified as

$$
S_{\eta}^{\left(\mathscr{R}_{p}\right)}(\delta)=\frac{1}{1-\eta} \operatorname{Ln} \frac{2 \pi}{\sigma} \int_{0}^{1} \delta(\varkappa)^{\eta} \frac{1}{1-\varkappa} d \varkappa
$$

where $\varkappa=1-e^{-2 \sigma r}$ and $\delta(\varkappa)$ denotes the probability density as

$$
\delta(\varkappa)=\mathscr{N}_{n}{ }^{2}(1-\varkappa)^{2 \zeta} \varkappa^{2 \rho}\left(\mathscr{P}_{n}^{(2 \zeta, 2 \rho-1)}(2 \varkappa-1)\right)^{2} .
$$

By inserting the probability density, Equation (23), into Equation (22), the Rényi entropy in the position space is modified as

$$
S_{\eta}^{\left(\mathscr{A}_{p}\right)}(\delta)=\frac{1}{1-\eta}\left(\operatorname{Ln} \frac{2 \pi}{\sigma}+\eta \operatorname{Ln} \frac{2^{2(\zeta+\rho+1)} \zeta \sigma(\rho+n+\zeta)(2 \rho+n) \Gamma(2 \zeta+n) \Gamma(2 \zeta+\rho+n+1)}{(n+\rho)(2 n+2 \rho+2 \zeta-1) \Gamma(2(\zeta+\rho)+n)}\right) .
$$

In order to obtain the corresponding Rényi entropy in the momentum space, one can take $t=1-2 e^{-2 \sigma r}$. Therefore, Equation (21) is more precisely written as

$$
S_{\eta}^{\left(\mathscr{R}_{m}\right)}(\gamma)=\frac{1}{1-\eta} \operatorname{Ln} \frac{2 \pi}{\sigma} \int_{-1}^{1} \gamma(t)^{\eta} \frac{1}{1-t} d t
$$




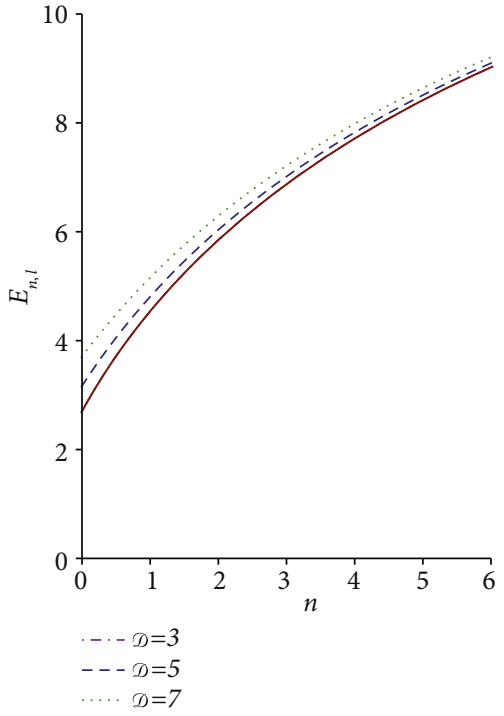

FIgURE 4: Variation of the energy $E_{n, \ell}$ in terms of $n$ with $\mu=c=\hbar$ $=1, H=20, \varrho=0.1, T=10$, and $\sigma=0.1$ for $\mathscr{D}=3,5$, and 7 .

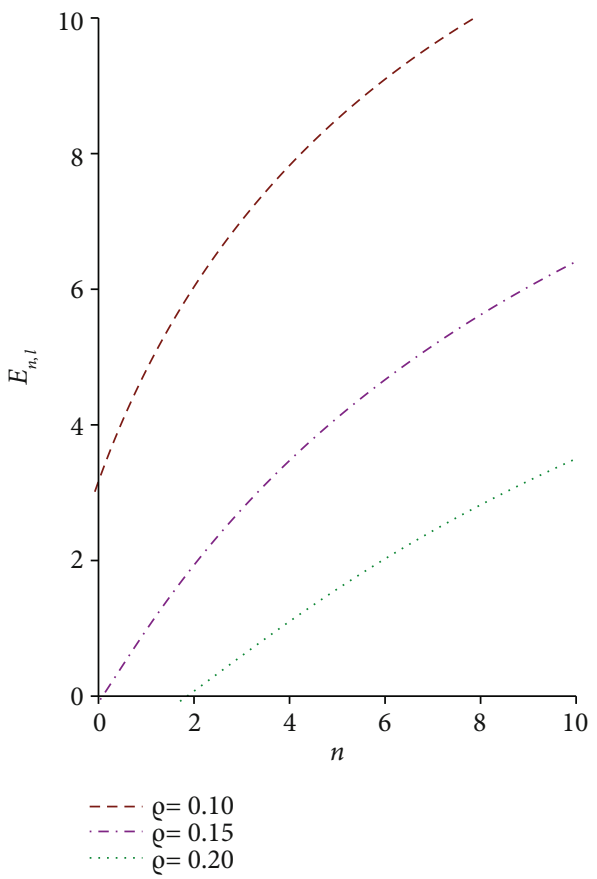

FIGURE 5: The function of energy spectral with regard to $n$ with $\mu$ $=c=\hbar=1, H=20, T=15, \mathscr{D}=3$, and $\sigma=0.1$ for $\mathrm{Q}=0.1,0.15$, and 0.20 .

By considering the appropriate probability density $\gamma(t)$, Equation (23), and substituting it into Equation (25), the compact form of the Rényi entropy in the momentum space is explicitly represented by

$S_{\eta}^{\left(\mathscr{R}_{m}\right)}(\gamma)=\frac{1}{1-\eta}\left(\operatorname{Ln} \frac{\pi}{\sigma}\left(\frac{4 \sigma(n+2 \rho)(\rho+n+\zeta) \Gamma(2 \zeta+n+1) \Gamma(2 \zeta+\rho+n+1)}{\Gamma(n+2 \rho+2 \zeta+1)(\rho+n)}\right)^{\eta}\right)$

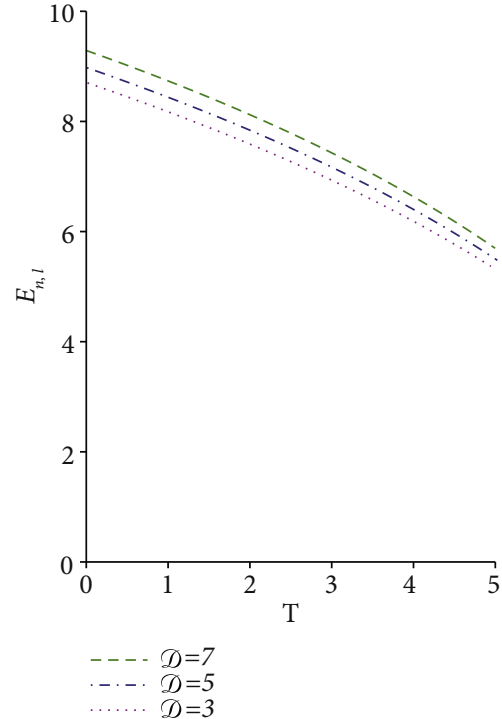

Figure 6: The contribution of the coupling strength to the energy spectra with $\mu=c=\hbar=1, H=10, \sigma=0.1$, and $\varrho=0.1$ for $\mathscr{D}=3$, 5 , and 7.

It is worth mentioning that when $\eta=1$, the Shannon entropy and Kullback-Leibler divergence are recovered [75].

4.2. Tsallis Entropy. In order to generalize the BoltzmannGibbs theory [76] and open the way of describing quantum subjects such as quantum optics and quantum communication [77], the Tsallis entropy was reported by Tsallis [75] in 1988. Tsallis entropy is often used in nonextensive systems, including nonergodic structures and processes; see, e.g., reference [73] for more details. By implementing the conversion $h=e^{-2 \sigma r}$, the Tsallis entropy becomes

$$
S_{\eta}^{\left(\mathscr{T}_{p}\right)}(\delta)=\frac{1}{\eta-1}\left(1-\frac{2 \pi}{\sigma} \int_{0}^{1} \delta(h)^{\eta} \frac{1}{h} d h\right)
$$

and is indicated in position space by

$$
S_{\eta}^{\left(\mathscr{T}_{p}\right)}(\delta)=\frac{1}{\eta-1}\left(1-\frac{2 \pi}{\sigma} \int_{0}^{1} \delta(\xi)^{\eta} \frac{1}{1-\varkappa} d z\right),
$$

where $\approx=1-e^{-2 \sigma r}$. By taking the probability density Equation (23) and inserting it into Equation (28), one can obtain the analogous entropy as

$$
\begin{aligned}
S_{\eta}^{\left(\mathscr{T}_{p}\right)}(\delta)= & \frac{1}{\eta-1}\left(1-\frac{2 \pi}{\sigma} \cdot\left(\frac{2^{2 \zeta+\rho+1} \sigma(n+\rho+\zeta)}{(n+\rho)(2 \zeta+2 \rho+2 n+1)}\right.\right. \\
& \left.\left.\cdot \frac{2 \zeta(n+2 \rho) \Gamma(2 \zeta+n) \Gamma(2 \zeta+\rho+n+1)}{\Gamma(2(\zeta+\rho)+n)}\right)^{\eta}\right) .
\end{aligned}
$$

Introducing $t=1-2 e^{-2 \sigma r}$ and substituting Equation (23) into Equation (27), the corresponding Tsallis entropy 


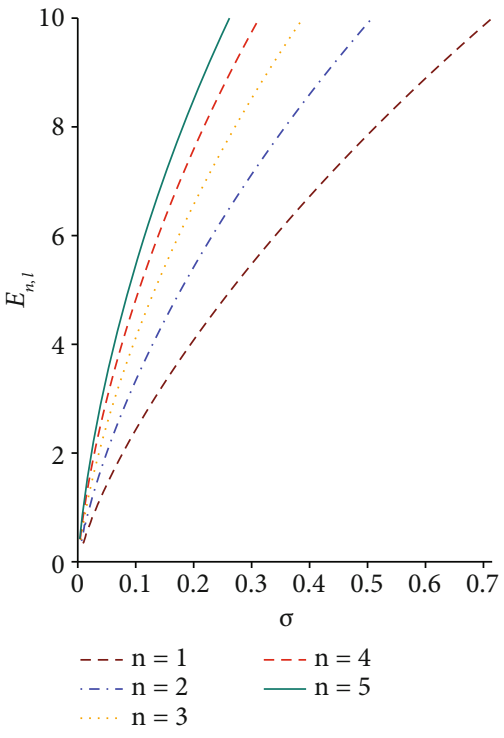

FIGURE 7: Contribution of the $\sigma$-parameters to the energy spectra with $\mu=c=\hbar=1, H=40, \mathscr{D}=3, T=0.5$, and $\mathrm{Q}=0.5$ for $n=1,2$, 3,4 , and 5 .

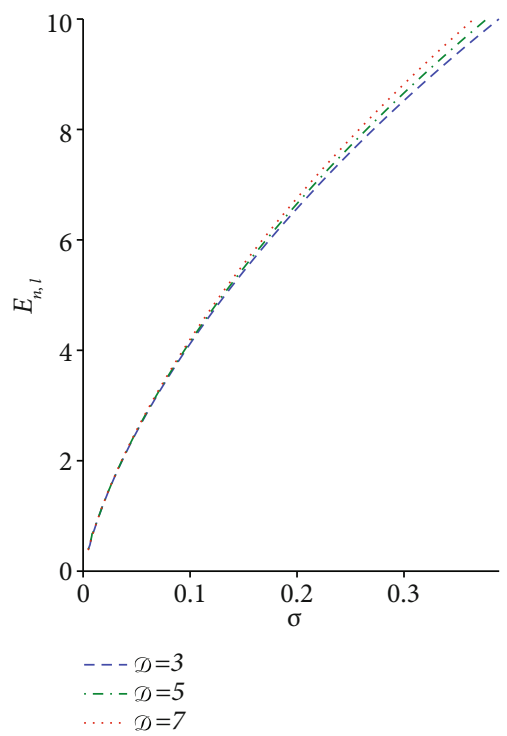

FIGURE 8: Effects of the screening parameter $\sigma$ on the energy spectra with $\mu=c=\hbar=1, H=40, T=0.5$, and $\mathrm{Q}=0.5$ for $\mathscr{D}=3$, 5 , and 7.

in the momentum space may further be represented as

$S_{\eta}^{\left(\widetilde{T}_{m}\right)}(\gamma)=\frac{1}{\eta-1}\left(1-\frac{\pi}{\sigma} \times\left(\frac{4 \sigma(n+2 \rho)(n+\rho+\zeta) \Gamma(2 \zeta+n+1) \Gamma(2 \zeta+\rho+n+1)}{(n+\rho) \Gamma(2 \zeta+2 \rho+n+1)}\right)^{\eta}\right)$.

It should be pointed out that, subject to the limit at $\eta=1$, one can resume the usual Boltzmann-Gibbs entropy [59].

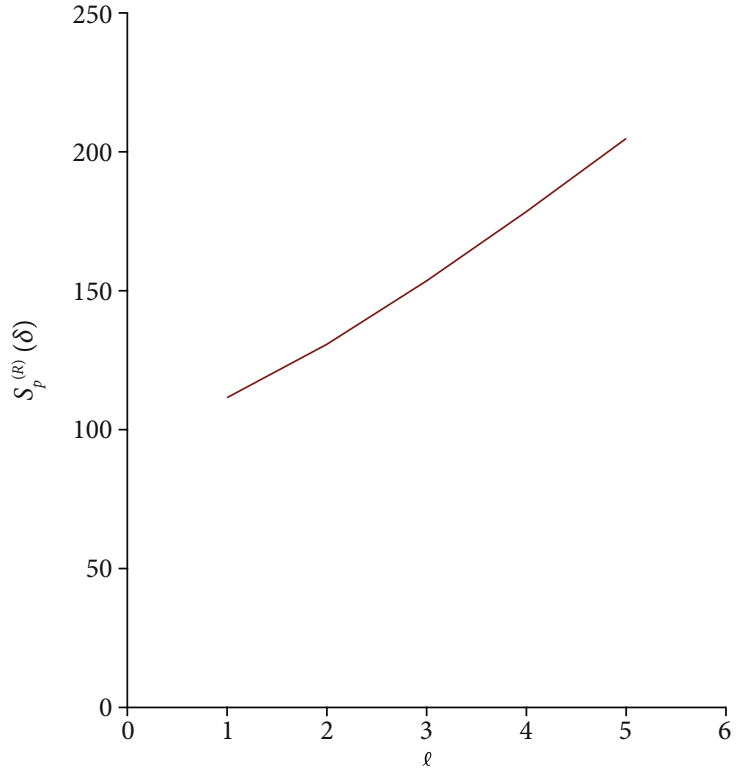

Figure 9: Behavior of the Rényi entropy for the position space $S_{p}^{(\mathscr{R})}(\delta)$ in terms of the angular momentum quantum number $\ell$ with $\mu=c=\hbar=1, H=20, T=10, \varrho=0.1$, and $\sigma=0.01$ for $\mathscr{D}=3$.

\section{Discussion}

Figure 2 depicts the variation of the generalized inversely quadratic Yukawa potential blended directly with the hyperbolic Schiöberg molecular potential in terms of internuclear separation with respect to the different screening parameters. It should be noted that around $r=1$, the figure decreases swiftly while for $r>1$, it gradually increases.

In Figure 1, the treatment of $1 / r^{2}$ and the suggested approximation are figured with different screening parameters, $\sigma$. The pattern tends to gather asymptotically for various values of the screening parameters, which serves as a good approximation of the system.

For the behavior of the energies in terms of the quantum number $n$ for different screening parameters, the dimension of the system and the potential molecular parameter are plotted, respectively, in Figures 3-5. In all cases, the energy curves increase as the quantum number increases. It can be observed that when each of the potential parameters increases, a molecule impacted by this type of potential exhibits almost identical behavior.

Figure 6 depicts the fluctuation of the energy eigenvalue with regard to the potential parameter $T$, where the energy of the system decreases monotonically with the increase in the potential parameter.

Figure 7 indicates the approximate energy $E_{n \ell}$, as far as the screening parameter, $\sigma$, within the proposed potential. It can be noticed that as the screening parameter, $\sigma$, expands, the approximate energy increases monotonically with increasing $n$.

It can be observed from Figure 8 that the energy increases smoothly with increasing $\sigma$ as the dimension of the system is increased $\mathscr{D}=3,5$, and 7 . 


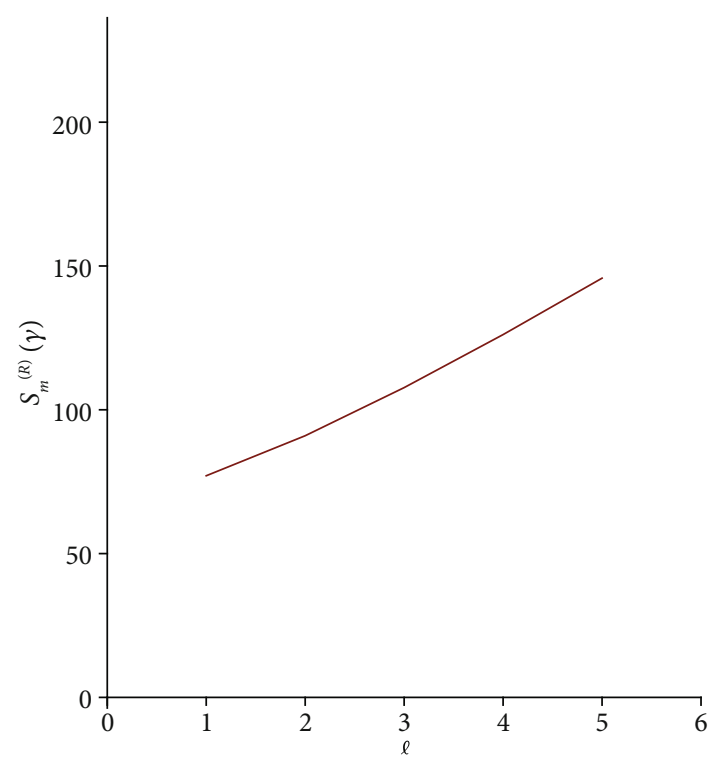

Figure 10: Variation of the Rényi entropy for the momentum space $S_{m}^{(\mathscr{R})}(\gamma)$ with respect to the angular momentum quantum number $\ell$ with $\mu=c=\hbar=1, H=20, T=10, \varrho=0.1$, and $\sigma=0.01$ for $\mathscr{D}=3$.

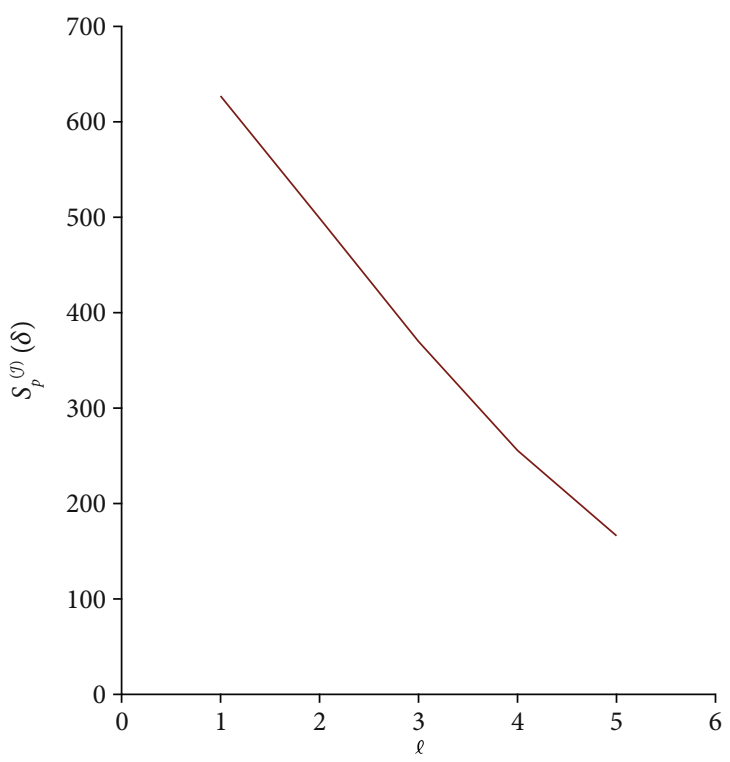

Figure 11: Effects of the Tsallis entropy for the position space $S_{p}^{(\mathscr{T})}(\delta)$ with respect to the angular momentum quantum number $\ell$ with $\mu=c=\hbar=1, H=20, T=10$, and $\mathrm{Q}=\sigma=0.1$ for $\mathscr{D}=3$.

Figures 9 and 10 illustrate the Rényi entropy in position space and momentum space in terms of the angular momentum quantum number for $\mathscr{D}=3$, respectively. In both cases, the Rényi entropy grows as the angular momentum quantum number increases.

It can be concluded from Figures 11 and 12 that the Tsallis entropy decreases with increasing angular momentum quantum number in both position space and momentum space.

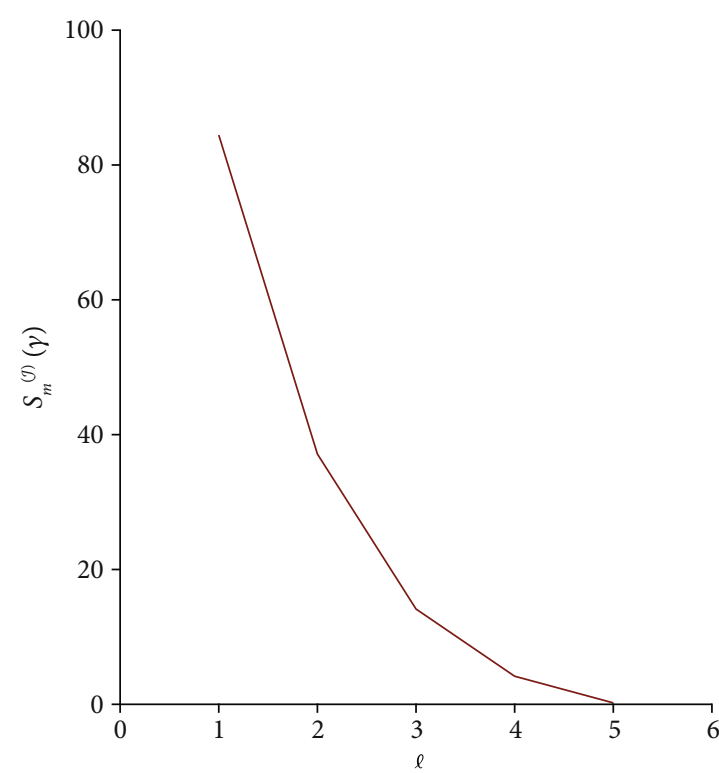

Figure 12: Contribution of the angular momentum quantum number $\ell$ to the Tsallis entropy for the momentum space $S_{m}^{(\mathscr{T})}(\gamma)$ with $\mu=c=\hbar=1, H=20, T=10$, and $\mathrm{Q}=\sigma=0.1$ for $\mathscr{D}=3$.

One can acquire from Table 1 that the energy eigenvalue in the nonrelativistic regime increases as the dimension of the system increments for practically all the states, while the energy turns out to be more negative in value and bounded as the screening parameter decreases in each state. It is fascinating to consider that the presented potential, Equation (1), shows some different highlights.

The peculiar cases of Equation (1), obtained by selecting the appropriate controlling parameters, can readily suggest distinguished interaction potential patterns. Based on this, one can realize that at $H=0$, and Equation (1) simplifies to the short-range generalized inversely quadratic Yukawa potential. This is regarded as a compelling model with applications in different branches of condensed matter and nuclear physics due to its ability to characterize the strong interaction between nucleons [78]. While the radial variable $r$ approaches to zero, it behaves similarly to a Yukawa potential, but the first term in R.H.S. in Equation (1) moderates its treatment at the origin. In this manner, the approximate analytical solutions of $\ell$-state $\mathscr{D}$-dimensional Klein-Gordon equation in the relativistic framework are obtained as

$$
\begin{aligned}
& 2 \sigma\left(n+\frac{1}{2}\right) \hbar c\left(4 \hbar^{2} c^{2}\left(\wp+\frac{1}{2}\right)^{2}-8 \mathrm{~T}\left(c^{2} \mu+E_{n, \ell}\right)\right) \\
& \quad+2\left(n+\frac{1}{2}\right) \hbar c\left(-\left(c^{2} \mu+E_{n, \ell}\right)\left(-c^{2} \mu+E_{n, \ell}+2 T\right)\right) \\
& +\left(\sigma \left(\frac{2 \sqrt{2}}{\sigma \hbar^{2} c^{2}} \sqrt{\left(\left(-\frac{(\wp+1 / 2)^{2} \hbar^{2}}{2}+T \mu\right) c^{2}+E_{n, \ell} T\right)\left(-c^{2} \mu+E_{n, \ell}+2 T\right)\left(c^{2} \mu+E_{n, \ell}\right)}\right.\right. \\
& \left.\left.+2\left((\wp+1) \wp+(n+1) n+\frac{1}{2}\right) \hbar^{2}+4 T \mu\right) c^{2}+4 E_{n, \ell} T\right)=0 .
\end{aligned}
$$

In particular, the results, when setting $H=0$, have good 
TABle 1: The energy spectra $E_{n, \ell}$ as a function of $\sigma$ with $\mu=c=\hbar$ $=1, H=20, T=10$, and $\mathrm{Q}=0.1$ for $\mathscr{D}=3,5$, and 7 .

\begin{tabular}{|c|c|c|c|c|}
\hline States & $\sigma$ & $\mathscr{D}=3$ & $\begin{array}{c}E_{n, \ell} \\
\mathscr{D}=5\end{array}$ & $\mathscr{D}=7$ \\
\hline \multirow{4}{*}{$2 p$} & 0.01 & -14.70968032 & -14.69313982 & -14.66835990 \\
\hline & 0.04 & -3.50480000 & -3.34087012 & -3.09988322 \\
\hline & 0.08 & 4.98981517 & 5.37429320 & 5.89847975 \\
\hline & 0.10 & 7.59500000 & 8.07647051 & 8.67994097 \\
\hline \multirow{4}{*}{$3 p$} & 0.01 & -13.98344446 & -13.96744208 & -13.94346812 \\
\hline & 0.04 & -1.83644800 & -1.69193926 & -1.47939292 \\
\hline & 0.08 & 6.63308671 & 6.93034652 & 7.33717322 \\
\hline & 0.10 & 9.02000000 & 9.36232471 & 9.79456521 \\
\hline \multirow{4}{*}{$3 d$} & 0.01 & -13.96744208 & -13.94346812 & -13.91155810 \\
\hline & 0.04 & -1.69193926 & -1.47939292 & -1.20359890 \\
\hline & 0.08 & 6.93034652 & 7.33717322 & 7.81732494 \\
\hline & 0.10 & 9.36232471 & 9.79456521 & 10.25681141 \\
\hline \multirow{4}{*}{$4 p$} & 0.01 & -13.28072216 & -13.26523528 & -13.24203342 \\
\hline & 0.04 & -0.36290651 & -0.23497208 & -0.04671569 \\
\hline & 0.08 & 7.91032605 & 8.14324976 & 8.46294660 \\
\hline & 0.10 & 10.03962810 & 10.28733674 & 10.60140386 \\
\hline \multirow{4}{*}{$4 ?$} & 0.01 & -13.26523528 & -13.24203342 & -13.21115064 \\
\hline & 0.04 & -0.23497208 & -0.04671569 & 0.19771695 \\
\hline & 0.08 & 8.14324976 & 8.46294660 & 8.84160634 \\
\hline & 0.10 & 10.28733674 & 10.60140386 & 10.93851446 \\
\hline \multirow{4}{*}{$4 ?$} & 0.01 & -13.24203342 & -13.21115064 & -13.17263208 \\
\hline & 0.04 & -0.04671569 & 0.19771695 & 0.49315365 \\
\hline & 0.08 & 8.46294660 & 8.84160634 & 9.25168299 \\
\hline & 0.10 & 10.60140386 & 10.93851446 & 11.26515817 \\
\hline
\end{tabular}

accuracy with those obtained in [53] in the nonrelativistic limit for $\mathscr{D}=3$.

However, when $H=0$ and in the low screening region where $\sigma \longrightarrow 0$, the Mie-type potential [79] is obtained, whose energy spectra, in accordance with Equation (31), are given by

$$
\begin{aligned}
& -\frac{1}{2 c \hbar \sqrt{c^{2} \mu-E_{n, \ell}-T}}\left(\hbar c\left(\sqrt{\frac{4 \hbar^{2} c^{2}(\wp+1 / 2)^{2}-4 T\left(c^{2} \mu+E_{n, \ell}\right)}{\hbar^{2} c^{2}}+2 n+1}\right)\right. \\
& \left.\times \sqrt{c^{2} \mu-E_{n, \ell}-T}+2 T \sqrt{c^{2} \mu+E_{n, \ell}}\right)=0 .
\end{aligned}
$$

In addition, the outcomes are in good agreement with those provided in [80], taking $\mathscr{D}=3$ in the nonrelativistic limit for the $\ell$-wave case, and they agree with those given in [81] for the Mie-type potential.

Setting $T=0$ yields the modified hyperbolic-type potential, suggested by Schiöberg as an important diatomic molecular potential [82]. This is strongly associated with the Morse [83], Kratzer [84], Coulomb [85, 86], harmonic oscillator $[87,88]$, and other potential functions as specific cases and is widely used in various applications such as quantum statistical theory [89], conformal field theory [90], nuclear structure [91], and chemical physics [92]. Thus, given this condition, the energy eigen-spectrum may be calculated as

$$
\begin{aligned}
& \frac{1}{2 c^{2} \hbar^{2} \sigma^{2}}\left(\left(\sqrt{c^{2} \mu+E_{n, \ell}} \sqrt{2(\varrho-1)^{2} H+c^{2} \mu-E_{n, \ell}}+2 \sigma\left(n+\frac{1}{2}\right) c \hbar\right)\right. \\
& \cdot \sqrt{8 \varrho^{2}\left(c^{2} \mu+E_{n, \ell}\right) H+4 \sigma^{2} c^{2} \hbar^{2}\left(\wp+\frac{1}{2}\right)^{2}+2 \hbar \sqrt{c^{2} \mu+E_{n, \ell}} \sigma\left(n+\frac{1}{2}\right) c} \\
& \cdot \sqrt{2(\varrho-1)^{2} H+c^{2} \mu-E_{n, \ell}+\left(2 \hbar^{2}\left(\wp^{2}+n^{2}+\wp+n+\frac{1}{2}\right) \sigma^{2}+4 H \mu \varrho(\varrho-1)\right) c^{2}} \\
& \left.+4 H E_{n, \ell} \mathrm{\varrho}(\varrho-1)\right)=0 .
\end{aligned}
$$

It is shown that the result given in this manner is consistent with the one obtained by Ortakaya in [93] for the Schrödinger equation within the hyperspherical coordinates.

\section{Conclusions}

The approximate $\ell$-state solutions for the $\mathscr{D}$-dimensional Klein-Gordon equation with a Schiöberg-generalized inversely quadratic Yukawa potential are investigated in this paper. The treatment of the respective energy eigenvalues is discussed as a function of various potential parameters. Particular samples of the potential and their analogous energy relations were concluded, which are compatible with those discussed in the literature. Two fundamental theoretic quantities, the Rényi entropy and the Tsallis entropy, are obtained which are useful in describing the synthetic reactivity of particles.

\section{Data Availability}

The calculated and acquired data employed to support the findings of this study are included in the article and are mentioned at appropriate positions within the manuscript as references.

\section{Conflicts of Interest}

The author declares that there is no conflict of interest regarding the publication of this paper.

\section{References}

[1] O. J. Oluwadare and K. J. Oyewumi, "The scattering phase shifts of the Hulthén-type potential plus Yukawa potential," The European Physical Journal Plus, vol. 131, no. 9, p. 295, 2016.

[2] M. C. Onyeaju, A. N. Ikot, E. O. Chukwuocha, H. P. Obong, S. Zare, and H. Hassanabadi, "Scattering and bound states of Klein-Gordon particle with Hylleraas potential within effective mass formalism," Few-Body Systems, vol. 57, no. 9, pp. 823831, 2016.

[3] C. A. Onate, M. C. Onyeaju, and A. N. Ikot, "Analytical solutions of the Dirac equation under Hellmann-Frost-Musulin potential,” Annals of Physics, vol. 375, pp. 239-250, 2016.

[4] C.-Y. Chen, D.-S. Sun, and F.-L. Lu, "Approximate analytical solutions of Klein-Gordon equation with Hulthén potentials for nonzero angular momentum," Physics Letters A, vol. 370, no. 3-4, pp. 219-221, 2007. 
[5] O. Bayrak, A. Soylu, and I. Boztosun, "The relativistic treatment of spin-0 particles under the rotating Morse oscillator," Journal of Mathematical Physics, vol. 51, no. 11, 2010.

[6] T. T. Ibrahim, K. J. Oyewumi, and S. M. Wyngaardt, "Analytical solution of N-dimensional Klein-Gordon and Dirac equations with Rosen-Morse potential," The European Physical Journal Plus, vol. 127, no. 9, p. 100, 2012.

[7] A. N. Ikot, H. P. Obong, I. O. Owate, M. C. Onyeaju, and H. Hassanabadi, "Scattering State of Klein-Gordon particles by q-parameter hyperbolic Poschl-Teller potential," Advances in High Energy Physics, vol. 2015, Article ID 632603, 7 pages, 2015.

[8] S. Guo-Hua and D. Shi-Hai, "Relativistic treatment of spinless particles subject to a Tietz-Wei oscillator," Communications in Theoretical Physics, vol. 58, no. 2, pp. 195-197, 2012.

[9] A. N. Ikot, O. A. Awoga, H. Hassanabadi, and E. Maghsoodi, "Analytical approximate solution of Schrödinger equation in $D$ dimensions with quadratic exponential-type potential for arbitraryl-state," Communications in Theoretical Physics, vol. 61, no. 4, pp. 457-463, 2014.

[10] R. L. Greene and C. Aldrich, "Variational wave functions for a screened Coulomb potential," Physical Review A, vol. 14, no. 6, pp. 2363-2366, 1976.

[11] B. J. Falaye, "Arbitrary $\ell$-state solutions of the hyperbolical potential by the asymptotic iteration method," Few-Body Systems, vol. 53, no. 3-4, pp. 557-562, 2012.

[12] A. K. Rey, "Studies on the bound-state spectrum of hyperbolic potential," Few-Body Systems, vol. 55, no. 2, pp. 143-150, 2014.

[13] J.-Y. Liu, G.-D. Zhang, and C.-S. Jia, "Calculation of the interaction potential energy curve and vibrational levels for the a3 $\mathrm{u}+$ state of Li27 molecule," Physics Letters A, vol. 377, no. 21-22, pp. 1444-1447, 2013.

[14] C.-S. Jia, T. Chen, and S. He, "Bound state solutions of the Klein-Gordon equation with the improved expression of the Manning-Rosen potential energy model," Physics Letters A, vol. 377, no. 9, pp. 682-686, 2013.

[15] B. Roy and R. Roychoudhury, "The shifted $1 / \mathrm{N}$ expansion and the energy eigenvalues of the Hulthen potential for 1 not $=0$," Journal of Physics A: Mathematical and General, vol. 20, no. 10, pp. 3051-3055, 1987.

[16] A. F. Nikiforov and V. B. Uvarov, Special Functions of Mathematical Physics, Springer, Boston, MA, 1988.

[17] C. Tezcan and R. Sever, "A general approach for the exact solution of the Schrödinger equation," International Journal of Theoretical Physics, vol. 48, no. 2, pp. 337-350, 2009.

[18] O. Bayrak, I. Boztosun, and H. Çiftci, "Exact analytical solutions to the Kratzer potential by the asymptotic iteration method," International Journal of Quantum Chemistry, vol. 107, no. 3, pp. 540-544, 2007.

[19] A. Stahlhofen and K. Bleuler, "An algebraic form of the factorization method," Il Nuovo Cimento B Series, vol. 104, no. 4, pp. 447-465, 1989.

[20] R. M. Edelstein, K. S. Govinder, and F. M. Mahomed, "Solution of ordinary differential equations via nonlocal transformations," Journal of Physics A: Mathematical and General, vol. 34, no. 6, pp. 1141-1152, 2008.

[21] B. J. Falaye, S. M. Ikhdair, and M. Hamzavi, "Formula method for bound state problems," Few-Body Systems, vol. 56, no. 1, pp. 63-78, 2015.

[22] A. N. Ikot, H. Hassanabadi, E. Maghsoodi, and S. Zarrinkamar, "D-dimensional Dirac equation for energy-dependent pseudo- harmonic and Mie-type potentials via SUSYQM," Communications in Theoretical Physics, vol. 61, no. 4, pp. 436-446, 2014.

[23] L. Fa-Lin, C. Chang-Yuan, and S. Dong-Sheng, "Bound states of Klein-Gordon equation for double ring-shaped oscillator scalar and vector potentials," Chinese Physics, vol. 14, no. 3, pp. 463-467, 2005.

[24] S.-H. Dong and Z.-Q. Ma, "Exact solutions to the Schrödinger equation for the potential in two dimensions," Journal of Physics A: Mathematical and General, vol. 31, no. 49, pp. 98559859, 1998.

[25] M. Roshanzamir-Nikou and H. Goudarzi, "The Laplace transform approach for a Dirac isotonic oscillator with a tensor potential in D-dimensions," Physica Scripta, vol. 89, no. 1, 2014.

[26] M. Roshanzamir-Nikou and H. Goudarzi, "Pauli isotonic oscillatorwith an anomalous magnetic moment in the presence of the Aharonov-Bohm effect: Laplace transform approach," Theoretical and Mathematical Physics, vol. 186, no. 2, pp. 286-293, 2016.

[27] H.-M. Tang, G.-C. Liang, L.-H. Zhang, F. Zhao, and C.-S. Jia, "Diatomic molecule energies of the modified Rosen-Morse potential energy model," Canadian Journal of Chemistry, vol. 92, no. 4, pp. 341-345, 2014.

[28] X.-T. Hu, L.-H. Zhang, and C.-S. Jia, "D-dimensional energies for cesium and sodium dimers," Canadian Journal of Chemistry, vol. 92, no. 5, pp. 386-391, 2014.

[29] W.-C. Qiang and S.-H. Dong, "Proper quantization rule," EPL (Europhysics Letters), vol. 89, no. 1, 2010.

[30] O. J. Oluwadare and K. J. Oyewumi, "Scattering state solutions of the Duffin-Kemmer-Petiau equation with the Varshni potential model," The European Physical Journal A, vol. 53, no. 2, p. 29, 2017.

[31] J. Jing, Y.-F. Zhang, K. Wang, Z.-W. Long, and S.-H. Dong, "On the time-dependent Aharonov-Bohm effect," Physics Letters B, vol. 774, pp. 87-90, 2017.

[32] Ö. Yeşiltaş, M. Şimşek, R. Sever, and C. Tezcan, "Exponential type complex and non-Hermitian potentials in PTsymmetric quantum mechanics," Physica Scripta, vol. 67, no. 6, pp. 472-475, 2003.

[33] M. Simsek and H. Egrifes, "The Klein-Gordon equation of generalized Hulthén potential in complex quantum mechanics," Journal of Physics A: Mathematical and General, vol. 37, no. 15, pp. 4379-4393, 2004.

[34] C. P. Onyenegecha, C. A. Onate, O. K. Echendu, A. A. Ibe, and H. Hassanabadi, "Solutions of Schrodinger equation for the modified Mobius square plus Kratzer potential," The European Physical Journal Plus, vol. 135, no. 3, 2020.

[35] N. Hatami, J. Naji, and M. Pananeh, "Analytical solutions of the Klein-Gordon equation for the deformed generalized Deng-Fan potential plus deformed Eckart potential," The European Physical Journal Plus, vol. 134, no. 3, p. 90, 2019.

[36] S.-H. Dong and M. Cruz-Irisson, "Energy spectrum for a modified Rosen-Morse potential solved by proper quantization rule and its thermodynamic properties," Journal of Mathematical Chemistry, vol. 50, no. 4, pp. 881-892, 2012.

[37] A. N. Ikot and I. O. Akpan, "Bound state solutions of the Schrödinger equation for a more general Woods-Saxon potential with arbitraryl-state," Chinese Physics Letters, vol. 29, no. 9, 2012.

[38] S. H. Dong, "Relativistic treatment of spinless particles subject to a rotating Deng-Fan oscillator," Communications in Theoretical Physics, vol. 55, no. 6, pp. 969-971, 2011. 
[39] K. J. Oyewumi, B. J. Falaye, C. A. Onate, O. J. Oluwadare, and W. A. Yahya, "Thermodynamic properties and the approximate solutions of the Schrödinger equation with the shifted Deng-Fan potential model," Molecular Physics, vol. 112, no. 1, pp. 127-141, 2014.

[40] S. M. Ikhdair and B. J. Falaye, "Approximate analytical solutions to relativistic and nonrelativistic Poschl- Teller potential with its thermodynamic properties," Chemical Physics, vol. 421, pp. 84-95, 2013.

[41] A. Suparmi, C. Cari, and B. N. Pratiwi, "Thermodynamics properties study of diatomic molecules with q-deformed modified Poschl-Teller plus Manning Rosen non-central potential in D dimensions using SUSYQM approach," Journal of Physics: Conference Series, vol. 710, 2016.

[42] J. J. Peña, J. García-Martínez, J. García-Ravelo, and J. Morales, "Bound state solutions ofD-dimensional schrödinger equation with exponential-type potentials," International Journal of Quantum Chemistry, vol. 115, no. 3, pp. 158-164, 2015.

[43] O. Aydoğdu and R. Sever, "Exact solution of the Dirac equation with the Mie-type potential under the pseudospin and spin symmetry limit," Annals of Physics, vol. 325, no. 2, pp. 373-383, 2010.

[44] W.-C. Qiang, R.-S. Zhou, and Y. Gao, "Application of the exact quantization rule to the relativistic solution of the rotational Morse potential with pseudospin symmetry," Journal of Physics A: Mathematical and Theoretical, vol. 40, no. 7, pp. 16771685, 2007.

[45] S. M. Ikhdair and R. Sever, "Solutions of the spatiallydependent mass Dirac equation with the spin and pseudospin symmetry for the Coulomb-like potential," Applied Mathematics and Computation, vol. 216, no. 2, pp. 545$555,2010$.

[46] L.-H. Zhang, X.-P. Li, and C.-S. Jia, “Approximate analytical solutions of the Dirac equation with the generalized Morse potential model in the presence of the spin symmetry and pseudo-spin symmetry," Physica Scripta, vol. 80, no. 3, 2009.

[47] S.-H. Dong, Factorization Method in Quantum Mechanics, Springer, Dordrecht, The Netherlands, 2007.

[48] A. Arda and R. Sever, "Exact solutions of the Morse-like potential, step-up and step-down operators via Laplace transform approach," Communications in Theoretical Physics, vol. 58, no. 1, pp. 27-30, 2012.

[49] A. Contreras-Astorga, D. J. Andez, and J. Negro, "Solutions of the Dirac equation in a magnetic field and intertwining operators," Symmetry, Integrability and Geometry: Methods and Applications (SIGMA), vol. 8, 2012.

[50] H. Feizi, M. R. Shojaei, and A. A. Rajabi, "Raising and lowering operators for the Dirac-Woods-Saxon potential in the presence of spin and pseudospin symmetry," The European Physical Journal Plus, vol. 127, no. 4, p. 41, 2012.

[51] A. Diaf and A. Chouchaoui, "Corrigendum: 1-states of the Manning-Rosen potential with an improved approximate scheme and Feynman path integral formalism," Physica Scripta, vol. 85, no. 3, 2012.

[52] M. R. Shojaei, A. A. Rajabi, M. Farrokh, and N. Zoghi-Foumani, "Energy levels of spin-1/2 particles with Yukawa interaction," Journal of Modern Physics, vol. 5, no. 9, pp. 773-780, 2014.

[53] B. J. Falaye, F. A. Serrano, and S.-H. Dong, "Fisher information for the position-dependent mass Schrodinger system," Physics Letters A, vol. 380, no. 1-2, pp. 267-271, 2016.
[54] W. Heisenberg, "Über den anschaulichen inhalt der quantentheoretischen kinematik und mechanik," Zeitschrift für Physik, vol. 43, no. 3-4, pp. 172-198, 1927.

[55] J. C. Angulo, "Information entropy and uncertainty inDdimensional many-body systems," Physical Review A, vol. 50, no. 1, pp. 311-313, 1994.

[56] A. Rényi, "On measures of information and entropy," in Proceedings of the 4th Berkeley Symposium on Mathematical Statistics and Probability, pp. 547-561, University of California Press: Berkeley, CA, USA, 1961.

[57] W. A. Yahya, K. J. Oyewumi, and K. D. Sen, "Quantum information entropies for the $\ell$-state Pöschl-Teller-type potential," Journal of Mathematical Chemistry, vol. 54, no. 9, pp. 18101821, 2016.

[58] S. H. Patil and K. D. Sen, "Scaling properties of net information measures for bound states of spherical model potentials confined with finite barrier," Journal of Chemical Sciences, vol. 124, no. 1, pp. 241-245, 2012.

[59] C. Tsallis, "Possible generalization of Boltzmann-Gibbs statistics," Journal of Statistical Physics, vol. 52, no. 1-2, pp. 479487, 1988.

[60] K. J. Oyewumi, "Analytical solutions of the Kratzer-Fues potential in an arbitrary number of dimensions," Foundations of Physics Letters, vol. 18, no. 1, pp. 75-84, 2005.

[61] S. M. Ikhdair and R. Sever, "Polynomial solutions of the Mietype potential in the $D$-dimensional Schrodinger equation," Journal of Molecular Structure: THEOCHEM, vol. 855, no. 13, pp. 13-17, 2008.

[62] I. S. Gradshteyn and I. M. Ryzhik, Table of Integrals, Series and Products, Academic Press, New York, NY, USA, 1965.

[63] A. N. Ikot, E. J. Ibanga, and H. Hassanabadi, "Scattering state of the multiparameter potential with an improved approximation for the centrifugal term in D-dimensions," International Journal of Quantum Chemistry, vol. 116, no. 2, pp. 81-87, 2016.

[64] Z.-W. Shui and C.-S. Jia, "Relativistic energies of the SiC radical in higher spatial dimensions," The European Physical Journal Plus, vol. 131, no. 6, 2016.

[65] A. Jeffrey and D. Zwillinger, Table of Integrals, Series, and Products, Academic, New York, 7th edition, 2007.

[66] J. S. Dehesa, S. López-Rosa, A. Martínez-Finkelshtein, and R. J. Yáñez, "Information theory of D-dimensional hydrogenic systems: application to circular and Rydberg states," International Journal of Quantum Chemistry, vol. 110, no. 8, pp. 1529-1548, 2010.

[67] G. Chechik, V. Sharma, U. Shalit, and S. Bengio, "Large scale online learning of image similarity through ranking," Journal of Machine Learning Research, vol. 11, pp. 1109-1135, 2010.

[68] I. V. Toranzo and J. S. Dehesa, "Rényi, Shannon and Tsallis entropies of Rydberg hydrogenic systems," EPL (Europhysics Letters), vol. 113, no. 4, p. 48003, 2016.

[69] Á. Nagy, "Fisher and Shannon information in orbital-free density functional theory," International Journal of Quantum Chemistry, vol. 115, no. 19, pp. 1392-1395, 2014.

[70] O. Olendski, "Rényi and Tsallis entropies: three analytic examples," European Journal of Physics, vol. 40, no. 2, article 025402, 2019.

[71] O. Gühne and M. Lewenstein, "Entropic uncertainty relations and entanglement," Physical Review A, vol. 70, no. 2, article 022316, 2004. 
[72] F. A. Bovino, G. Castagnoli, A. Ekert, P. Horodecki, C. M. Alves, and A. V. Sergienko, "Direct measurement of nonlinear properties of bipartite quantum states," Physical Review Letters, vol. 95, no. 24, article 240407, 2005.

[73] B. Kraus, N. Gisin, and R. Renner, "Lower and upper bounds on the secret-key rate for quantum key distribution protocols using one-way classical communication," Physical Review Letters, vol. 95, no. 8, article 080501, 2005.

[74] C. Beck and D. Graudenz, "Symbolic dynamics of successive quantum-mechanical measurements," Physical Review A, vol. 46, no. 10, pp. 6265-6276, 1992.

[75] T. Van Erven and P. Harremoes, "Rényi divergence and Kullback-Leibler divergence," IEEE Transactions on Information Theory, vol. 60, no. 7, pp. 3797-3820, 2014.

[76] L. S. F. Olavo, A. F. Bakuzis, and R. Q. Amilcar, "Generalized Schrodinger equation using Tsallis entropy," Physica A: Statistical Mechanics and its Applications, vol. 271, no. 3-4, pp. 303323, 1999.

[77] C. Tsallis, Introduction to Nonextensive Statistical Mechanics, Springer, New York, 2008.

[78] S. M. Ikhdair, M. Hamzavi, and B. J. Falaye, "Relativistic symmetries in Yukawa-type interactions with Coulomb-like tensor," Applied Mathematics and Computation, vol. 225, pp. 775-786, 2013.

[79] G. Mie, "Zur kinetischen theorie der einatomigen körper," Annalen der Physik, vol. 316, no. 8, pp. 657-697, 1903.

[80] L. Hitler, B. I. Ita, P. A. Isa et al., "Wkb solutions for inversely quadratic Yukawa plus inversely quadratic Hellmann potential," World Journal of Applied Physics, vol. 2, no. 4, pp. 109112, 2017.

[81] S. Miraboutalebi, "Solutions of Klein-Gordon equation with Mie-type potential via the Laplace transforms," The European Physical Journal Plus, vol. 135, no. 1, p. 16, 2020.

[82] D. Schiöberg, "The energy eigenvalues of hyperbolical potential functions," Molecular Physics, vol. 25, no. 8, pp. 11231137, 1986.

[83] H.-S. Sun, "Quantization rule for relativistic Klein-Gordon equation," Bulletin of the Korean Chemical Society, vol. 32, no. 12, pp. 4233-4238, 2011.

[84] A. Kratzer, "Die ultraroten rotationsspektren der halogenwasserstoffe," Zeitschrift für Physik, vol. 3, no. 5, pp. 289-307, 1920.

[85] O. L. De Lange, "An operator analysis for the Schrödinger, Klein-Gordon, and Dirac equations with a Coulomb potential," Journal of Mathematical Physics, vol. 30, no. 4, pp. 858866, 1989.

[86] S. Dong and S.-H. Dong, "Schrödinger equation with a Coulomb field in 2+1 dimensions," Physica Scripta, vol. 66, no. 5, pp. 342-344, 2002.

[87] D. Faiman and A. W. Hendry, "Harmonic-oscillator model for baryons," Physical Review, vol. 180, no. 5, pp. 1609-1610, 1969.

[88] L. Brey, N. F. Johnson, and B. I. Halperin, "Optical and magneto-optical absorption in parabolic quantum wells," Physical Review B, vol. 40, no. 15, pp. 10647-10649, 1989.

[89] H. J. De Vega, "Yang-Baxter algebras, integrable theories and quantum groups," International Journal of Modern Physics A, vol. 4, no. 10, pp. 2371-2463, 1989.

[90] A. Algin, M. Arik, and A. S. Arikan, "High temperature behavior of a two-parameter deformed quantum group fermion gas," Physical Review E, vol. 65, no. 2, article 026140, 2002.
[91] A. Ballesteros, O. Civitarese, and M. Reboiro, "Correspondence between theq-deformed harmonic oscillator and finite range potentials," Physical Review C, vol. 68, no. 4, article 044307, 2003.

[92] Y. Sun, S. He, and C.-S. Jia, "Equivalence of the deformed modified Rosen-Morse potential energy model and the Tietz potential energy model," Physica Scripta, vol. 87, no. 2, article 025301, 2013.

[93] S. Ortakaya, "Nonrelativistic $\ell$-state solutions for Schiöberg molecular potential in hyperspherical coordinates," Few-Body Systems, vol. 54, no. 11, pp. 1901-1909, 2013. 